\title{
Forecasting Tourist Arrivals and Supply and Demand Gap Analysis for Hotel Sector in Addis Ababa, Ethiopia
}

\author{
Samuel Liben ${ }^{1}$ Matiwos Ensermu ${ }^{2}$
}

\begin{abstract}
This paper aims to forecast the long term behavior of tourist arrivals and analyze the gap between supply and demand for the hotel/accommodation sector of the city of Addis Ababa. It also intends to provide vital information in regards to the sparse knowledge in the subject of forecasting tourist arrivals in Ethiopia. The research is largely conducted based on the secondary data obtained from the Ministry of Culture and Tourism (MOCT) in the tenth edition of the Policy, Planning, Evaluation and Monitoring Directorate's bulletin publication on Tourism Statistics (2009-2012),(MOCT, 2013). Theoretical assessments of the requirement of a forecasting process and a critical analysis of available forecasting methods have been carried out to fit the profile of long term tourist arrivals. Based on the assessments and analysis, the Box-Jenkins process was selected. Furthermore, the gap analysis is done using the Funneling Technique. The method has also determined that the annual tourist arrivals for the country in the year 2015 will be 798,157 and for the year of 2020, it is expected to be 1,130,971 and finally in 2025, the annual tourist arrivals are expected to climb to 1,463,743. The use of the Funneling Technique in combination with the Stepped Function Intervention Model establishes a different case scenario (positive, negative and starched intervention) which has then been studied to foresee the relationship between supply and demand of the accommodation sector under different circumstances.
\end{abstract}

Keywords: Forecasting, tourist arrival, X-12-ARIMA, Supply, demand.

http://dx.doi.org/10.4314/ejbe.v5i2.5

\footnotetext{
${ }^{1}$ MBA, Chief Engineer, Hilton Addis Ababa, e: mail: Libensamuel@gmail.com; Samuel.liben@hilton.com ${ }^{2} \mathrm{PhD}$, Associate Professor, Addis Ababa University, College of Business and Economics, email: ensermujalata@gmail.com
} 
Forecasting Tourist Arrivals and Supply and Demand Gap Analysis

\section{Introduction}

Tourism is an economic activity of primary value and importance for many countries. It is increasingly being recognized as a valued mechanism for job creation, economic development and poverty reduction. As a result, it is being promoted as an important source of economic growth especially in developing countries. Over the past decades, tourism has experienced a continued expansion and diversification, hence becoming one of the largest and rapidly growing economic sectors in the world (UNTWO, 2013). UNTWO's publication on tourism, towards 2030(UNWTO, 2012), shows that tourism offers a great potential for further expansion in coming decades. Emerging as well as established destinations can benefit from this opportunity, provided that they have adequate conditions and policies with regards to business environment, infrastructure, facilitation, marketing and human resources. Christie and Crompton in their paper have indicted that "The quality of Africa's resource endowment for tourism is exceptional, but most countries have only barely developed their tourism potential." (Christie \& Crompton, 2001). The continent receives about 5\% of all international travelers and tourism receipts (UNTWO, 2012), but tourism is 'significant' (>2\% of GDP and $>5 \%$ of exports) in about half of the countries found in Sub-Saharan Africa (SSA). Countries in Africa are now focusing on tourism as a source of growth and diversification. In 2013, Africa had attracted an additional 5\% of international tourists which corresponds to 3 million new arrivals. Over all, the region received 56 million tourists which translate to $5 \%$ of the tourists in the world. North Africa received more than $6 \%$ while Sub-Saharan Africa is estimated to have received 5\% (UNTWO, 2014).

Ethiopia is the site of some of the oldest human settlements in Africa. Recorded history in Ethiopia began around $1000 \mathrm{BC}$, in the time of King Solomon and the Queen of Sheba. However, recently discoveries indicate the existence of a rich prehistory. Evidence of Ethiopia's culture and history can be found in its ancient monuments, cities, and prehistoric sites while its intangible culture is reflected in the work of local architects, musicians, writers, artisans and craftsmen (Christie \& Crompton, 2001). The country has abundant heritage and tourism resources for which it has been globally highly ranked at the $38^{\text {th }}$ position, at par with Egypt which is well known globally for its predominantly heritage based tourism resources, including the majestic pyramids. Despite the high ranking of both destinations in 2008, Egypt has had more arrivals than Ethiopia. For instance, there were 12.2 million international tourist arrivals in Egypt compared to the 330,000 in Ethiopia (UNTWO, 2012).

EJBE Vol. 5 No. 2/2015

Page 270 
Forecasting Tourist Arrivals and Supply and Demand Gap Analysis

Although other factors could explain the difference in arrivals, the deduction alone suggests a great potential for heritage tourism development in Ethiopia.

\subsection{Background: Ethiopian Tourism Sector Performance}

Ethiopia's tourism sector is surely growing from the low base of a minimal $1 \%$ share of Africa's tourism market, but it continues to underperform despite its hidden potential with just a total of 596,341 tourist arrivals in 2012 (WBG \& MOCT 2012). Despite economic imbalances, such as the high inflation in previous years and limited access to financing in the private sector, Ethiopia's tourism sector has been showing a positive trend and a steady growth in tourism arrivals and receipts (WBG \& MOCT, 2012). The industry has emerged as one of the leading sectors that have the potential of driving the Ethiopian economy. It is now the third main source of foreign exchange earnings in Ethiopia (International Visitor's Survey, MOCT, 2013). Ethiopia's National Growth and Transformation Plan (GTP), active from 2010 to 2015, has set ambitious goals for the tourism sector. Such goals include the increase of current tourism arrivals to 1 million and a twelve fold increase in tourists' expenditures from 250 million US\$ in 2010 to 3 billion US\$ by 2015. However, the target seems a bit illusive to be realized within the set time period. The current government sees the sector as a priority and is in the process of revising the overall strategic plan. It has also established a new institution called Ethiopian Tourist Organization fully dedicated to oversee and coordinate the sector's activity.

Ethiopia's comparative advantage as a tourist destination is based on offering a safe, unspoiled, and a relatively unexplored 'Africa in one country'. With Addis Ababa serving as a diplomatic capital and a regional hub for air transport while the country itself offers attractive complementarities with neighboring destinations that possess marketability such as Kenya and Tanzania (WBG \& MOCT ;2012).

\subsection{Problem Statement}

The main problem to be addressed here revolves around the lack of information regarding the projection of future tourist (visitor) arrivals. In the current situation, it is difficult to have an idea or an estimate of arrivals for this year or the year after, neither there is an idea about the expected arrivals for the next five years. What is available thus far is the total number of arrivals from past years. However, such information, at best, is historic. Data of this kind can only provide limited insight. It does not determine the expected volume of 
Forecasting Tourist Arrivals and Supply and Demand Gap Analysis

arrivals for a future period. This information can only show the trend of the present situation and is unable to provide the necessary level of detail for decision making on investments in destination infrastructure which requires a long term financial commitment. Furthermore, it is also less than sufficient for policymaking.

Conflicting arguments and recommendation exist regarding supply and demand gaps. For example, a case study for a major international hotel chain indicates that it is challenging to get enough information on the sector, but based on what is available, they have concluded that there is an excess supply of rooms in Addis Ababa. Whereas other reports claim otherwise, the second opinion argues that there is a shortage of hotel rooms. MOCT (2009),Gobena\&Gudeta (2013) report of multi-link consult for long stay at Marriott Apartment Hotel set up in Addis Ababa.

There is also ample evidence in the city that points to investors looking for hotel development sites. This shows that investors believe that the market has business potential. Significant amounts of resources are invested based on assumptions or personal opinions with a less than satisfactory probing of the market situation.

\subsection{Research Question}

The basic research questions are: 'What will be the expected amount of tourist arrivals for the country as opposed to the city of Addis Ababa for the next five to ten years?' and 'What is the supply and demand relationship of the accommodation sector in Addis Ababa?'

The objectives are:

- To study and forecast visitors arrival (Tourist Arrival; TA) to the City of Addis Ababa.

- Since Addis Ababa is the main hub of the inbound \& out bound travel, the forecast could also hold for the country. Therefore the forecast can provide or fill the vital information gap for tourism especially for the hospitality sector in the country by providing Tourist Arrival (TA), for longer period.

- To predict long-term demand for tourism that will be critical input for part of the investment project appraisals for tourism related infrastructure such as hotels, roads, air ports.

- To make accurate forecasts of demand in the tourism sector of the economy which will help policy makers in formulating and implementing appropriate medium to long term policy and strategy.

EJBE Vol. 5 No. 2/2015 
Forecasting Tourist Arrivals and Supply and Demand Gap Analysis

- Based on the long range forecast analysis, to find out the trend of demand for hotel rooms in Addis Ababa.

- Based on the demand analysis to find out the gap between the supply \& demand for hotel rooms in Addis Ababa

\section{Review of Related Literature}

\subsection{Overview of International Tourism}

The United Nations World Tourism Organization (UNWTO) on its update of the long term outlook, Tourism Towards 2030 has indicated the future tourism trends. Growth is reported at a moderate pace in comparison to the previous estimate; it will slow down from $4.2 \%$ per year (1980-2000) to $3.3 \%$ per year (2010-2030). The growth represents some 43 million more international tourist arrivals every year, reaching a total of 1.8 billion arrivals by 2030. At the same time, UNWTO also announced that the total export earnings generated by international tourism in 2013 was 1.4 trillion US\$. Receipts earned by destinations from international tourists grew by $5 \%$ to reach 1159 billion US\$ while an additional 218 billion US\$ was earned by international passenger transport. International tourism, including travel and transport, accounts for $29 \%$ of the world's Export of service and $6 \%$ of the overall export of goods and services.

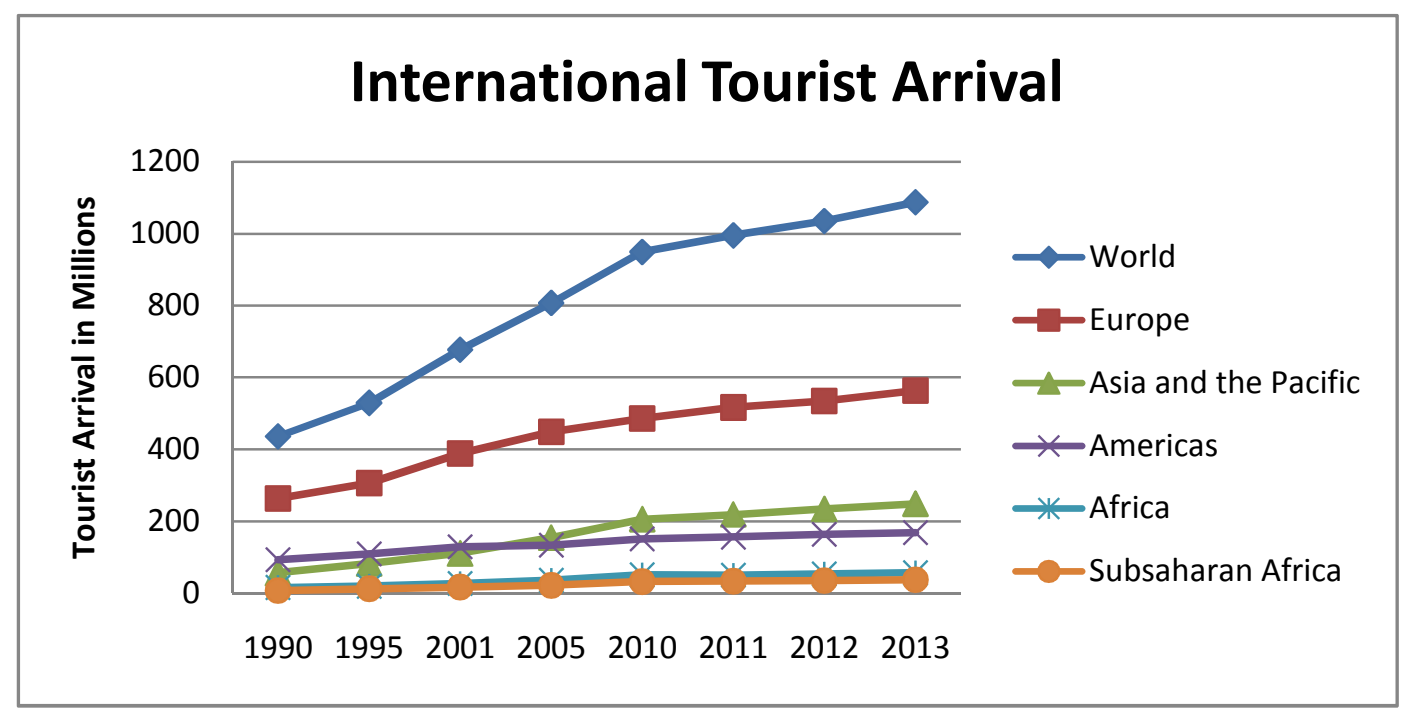

Fig 2-1: International Tourist Arrival. Source: UNTWO, 2014.

EJBE Vol. 5 No. 2/2015

Page 273 
Forecasting Tourist Arrivals and Supply and Demand Gap Analysis

Europe is the main tourist generating country in terms of population; Asia, Pacific, America, Middle East and Africa follow suit. On the other hand, Europe still has the highest inbound market share. However, the market share for Europe and America show a declining trend while other regions like Asia and Pacific grow faster (UNTWO, 2012). With regards to long term forecast, UNTWO's Tourism Highlight 2014 has reconfirmed the earlier long term forecast on Tourism Towards 2030 by upholding the claim that the international tourist arrival is expected to increase by $3.3 \%$ per year. It also indicates that from 2010 to 2030, arrivals in emerging destinations are expected to increase by $4.4 \%$ each year with an increase of twice the rate for those with advanced economies $(+2.2 \%$ a year). The market share of emerging economies increased from $30 \%$ in 1980 to $47 \%$ in 2013 and is expected to reach $57 \%$ by 2030, an equivalent to over 1 billion international tourist arrivals (UNTWO, 2014). UNTWO revised the growth of international tourist arrivals for 2014 to $4 \%-4.5 \%$ from the 3.3\% indicated by Tourism towards 2030 and later on confirmed that the international tourist arrivals for 2014 reached 1, 138 million. This is 51 million more than the amount of visitors in 2013, a $4.7 \%$ increase. It also indicates that the forecast of the international tourist arrivals of 2015 is expected to grow by 3\% to 4\% (UNTWO, 2015).

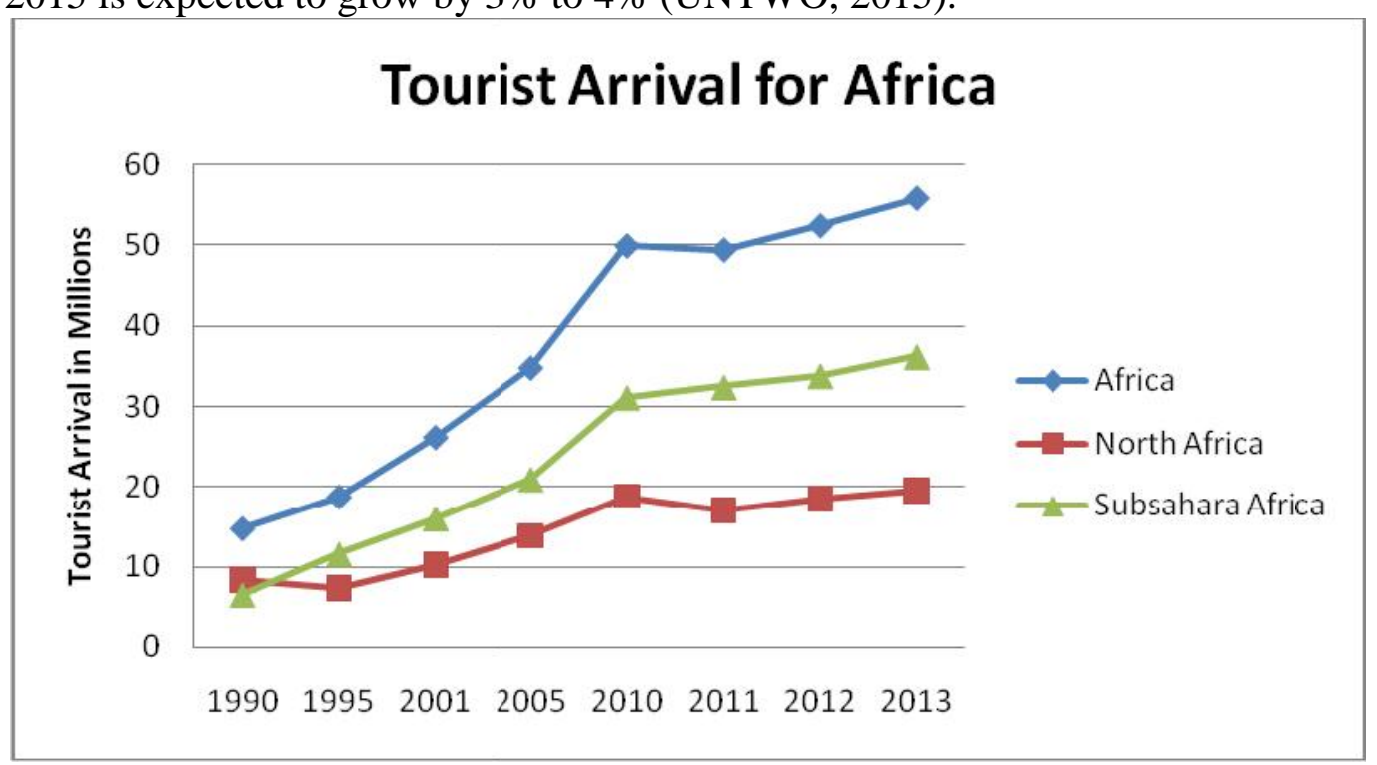

Fig 2-2: Tourist Arrival for Africa. Source: UNTWO, 2014.

\subsection{Tourism in Africa}

As cited by Idowk. $\mathrm{K}$, "Tourism in the African continent has been seen as a means of enhancing economic growth and development (Kester, 2003) as well 
Forecasting Tourist Arrivals and Supply and Demand Gap Analysis

as launching the image of the continent to the outside world. African countries have started to tap the potential that is embedded in tourism and hospitalities. According to Kester, tourism has the potential to contribute significantly to economic growth and development in Africa (2003). Naude and Saayman opined that Africa's cultural and natural resource endowments are such that it ought to be benefiting largely from tourism (2004)."Christie and Crompton believe that Africa has "exceptional" tourism potentials and that it is increasingly contributing to the continent's gross domestic products (GDP) and exports (Christie \& Crompton, 2001).

\subsection{Ethiopia's Tourism Attraction}

Ethiopia is well known for its diverse historical, cultural, and natural attractions. Historically, Ethiopia is quite ancient, having three thousand years worth of history. The great stales of Axum prove that the Axumite Empire was one of the greatest civilizations of the ancient world. The Rock-hewn churches of Lalibela show the religious civilization and devotion of the late middle ages. The majestic $17^{\text {th }}$ century castles of Gonder hint at the historic wealth of the country. Culturally, Ethiopia is teeming with diverse ethnic groups which have their own religion, language, culture, and tradition. With regards to the gifts of nature, Ethiopia offers breathtaking sceneries; embracing all kinds of relief, from the tops of the rugged Semien Mountains to the depths of the Danakil Depression which is more than 100 meters below sea level. In addition to that, Ethiopia is home to the fourth largest zone of biodiversity; it houses numerous unique floras more than any country in Africa. Among the abundant species of fauna and flora, 31 mammals, 30 amphibians, 17 birds, 14 reptiles, 4 fish and about 1000 plant species are endemic to Ethiopia.

It is probably the only country in Africa that hosts nine world heritage sites. The most prominent site being the Ethiopian Rift Valley; it is the site of many fossil findings that attest to it being the 'cradle of mankind' where humans started to walk upright. On top of all of that, its people are very hospitable and their cultures are diversified, a combination that is very suitable for tourism development. It is a place where nature, culture, and history merge to form a timeless appeal. (MOCT, Ethiopia: A Tourist Paradise).

\subsection{Importance of Forecasting for the Tourism Industry}

Forecasting tourism demand is important for tourism planning at all levels in the tourism industry from the government to a single tourist business (Cuhadar.M, 2014). Effective forecasting provides credible and timely 
Forecasting Tourist Arrivals and Supply and Demand Gap Analysis

information for tourism managers to balance the market demand with the tourism supply.The value of forecasting lies in its ability to reduce the loss caused by disparities between demand and supply.In order to provide satisfactory services to tourists, destinations need to acquire reliable forecasts of future demand. Accurate forcast of tourism demand are paramount to ensure availability of such service whereas lack of knowledge on tourist arrivals may lead to missed opportunities or an overestimation of tourism demand (A saayman\& M.Saayman, 2013). Overestimating tourism demand may, for instance, lead to excessive investment. Thus, forecasting is an integral part of the overall strategic planning process in the tourism industry.

Forecasting is also highly important for the tourism industry which needs accurate predictions of demand so that it can plan effectively from season to season, year to year.This, in turn, improves the efficiency of businesses, increase profits and strengthens economies.

\subsection{Forecasting Methods Selection}

UNWTO's report indicates that over the past decades tourism has experienced continuous expansion and diversification. This growth and expansion has generated an interest in tourism forecasting. Accordingly, a number of articles were published on tourism demand forecasting. Armstrong has published some general guidelines to select forecasting methods. These guidelines comprise many factors some of which are: convenience, market popularity, structured judgment, statistical criteria, relative track records and guidelines from prior research (Armstrong, 2001). Some checklists for selecting the best forecasting method in a given situation are also recommended as guidelines for selecting forecasting methods (Wang etal, 2006). Fretchling has discussed a model selection procedure; he proposed preliminary selection criteria to narrow the list of available methods down to a few key approaches (Fretchling .D.G 2001).

From both selecting procedures, Armstrong as well as Fretchling, it is possible to deduct that for a time series data such as tourist arrivals, the recommended forecasting method is the Quantitative method specifically the Extrapolation method. Time series forecasting are also called the extrapolative methods, because they extrapolate from the previous data in the series to predict future trends. These models attempt to identify the patterns in the time series that cause shifts in the forecast variable and to see how they interact. The advantage of such models is that they are relatively simple to estimate, requiring no more than one data series (Peng Bo, 2012).

EJBE Vol. 5 No. 2/2015

Page 276 
Forecasting Tourist Arrivals and Supply and Demand Gap Analysis

However, Extrapolation by itself is no single method it is a comprehensive name for different methods which are applicable to time series. It covers: Naïve, Single Moving Average, Single Exponential Smoothing, Double Exponential Smoothing and Autoregressive.

\section{Time Series Methods}

\begin{tabular}{|c|c|c|c|}
\hline No & $\begin{array}{c}\text { Forecasting } \\
\text { Method }\end{array}$ & Equation & Legend \\
\hline 1 & Naïve & $\begin{array}{l}\mathrm{F}_{\mathrm{t}}=\mathrm{At}_{1} \ldots \ldots \ldots \ldots \ldots \ldots \ldots \ldots \ldots \ldots \ldots \\
(1) \\
\mathrm{F}_{\mathrm{t}}=\mathrm{A}_{\mathrm{t}-1} *\left[1+\left[\left(\frac{A_{t-1}-A_{t-2}}{A_{t-2}}\right)\right] \ldots\right. \\
(2) \\
\mathrm{F}_{\mathrm{t}}=\boldsymbol{A}_{t-m} \ldots \ldots \ldots \ldots \ldots \ldots \ldots \ldots \ldots \\
(3)\end{array}$ & $\begin{array}{l}\text { Where: } \\
F=\text { forecast value } \\
A=\text { actual value } \\
t=\text { some time period } \\
m=\text { number of periods in } \\
\text { a year }\end{array}$ \\
\hline 2 & $\begin{array}{l}\text { Simple } \\
\text { moving } \\
\text { Average } \\
\text { (SMA) }\end{array}$ & $\mathrm{F}_{\mathrm{t}}=\left(\mathrm{A}_{\mathrm{t}-1}+\mathrm{A}_{\mathrm{t}-2}+\ldots \mathrm{A}_{\mathrm{t}-\mathrm{n}}\right) / \mathrm{n}$ & $\begin{array}{l}\text { Where: } \\
F=\text { forecast value } \\
A=\text { actual value } \\
t=\text { some time period } \\
n=\text { number of past time } \\
\text { periods }\end{array}$ \\
\hline 3 & $\begin{array}{l}\text { Single } \\
\text { Exponential } \\
\text { smoothing } \\
\text { (SES) }\end{array}$ & $\mathrm{F}_{\mathrm{t}}=\alpha \times \mathrm{A}_{\mathrm{t}-1}+(1-\alpha) \times \mathrm{F}_{\mathrm{t}-1}$ & $\begin{array}{l}\text { Where: } \\
F=\text { forecast value } \\
\mathrm{A}=\text { actual value } \\
\mathrm{t}=\text { some time period } \\
\alpha=\text { smoothing constant } \\
\text { between } 0 \text { and } 1\end{array}$ \\
\hline 4 & $\begin{array}{l}\text { Double } \\
\text { exponentials } \\
\text { moothing } \\
\text { (Brown`s } \\
\text { DES) }\end{array}$ & $\begin{array}{l}Y_{t}=\alpha A_{t-1}+(1-\alpha) Y_{t-1} \\
Y_{t}=\alpha Y_{t-1}+(1-\alpha) Y_{t-1}^{\prime} \\
C_{t}=Y_{t}+\left(Y_{t}-Y_{t}\right) \\
T_{t}=[(1-\alpha) / \alpha]^{*}\left(Y_{t}-Y_{t}\right) \\
F_{t}+n=C_{t}+n^{*} T_{t}\end{array}$ & $\begin{array}{l}\text { Where } \\
\mathrm{F}_{\mathrm{t}}=\text { Forecast at time } \mathrm{t} \text { : } \\
\mathrm{A}_{\mathrm{t}}=\text { actual value at time } \mathrm{t} \text {; } \\
\mathrm{Y}_{\mathrm{t}}=\text { SES seriesat time } \mathrm{t} ; \\
\mathrm{Y}^{`} \mathrm{t}=\mathrm{DES} \text { series at time } \mathrm{t} ; \\
\mathrm{C}_{\mathrm{t}}=\text { the intercept; } \\
\mathrm{T}_{\mathrm{t}}=\text { the slope coefficient; } \\
\mathrm{n}=\text { the number of forecast } \\
\text { period; }\end{array}$ \\
\hline
\end{tabular}

Naïve

EJBE Vol. 5 No. 2/2015 
Forecasting Tourist Arrivals and Supply and Demand Gap Analysis

No-change models are used very frequently in tourism demand forecasting and, surprisingly, they often give the most accurate calculations. (UNWTO\& ETC, 2008)

The Naive 1 (or no change) model is the simplest forecasting method for the period $\mathbf{t}$ is equal to the actual value in the last period (t-1).The Naive 2 model is version of Naïve which is widely used but a simple model employed when there is a continuous trend present in the data. The forecast value for period $\mathbf{t}$ is obtained by multiplying the demand over period t-1 by the growth rate between the period t-2 and the current period (t- 1). It assumes that growth rates remain unchanged from one time period to the next. (UNWTO \& ETC, 2008)

\section{Simple Moving Average (SMA)}

The SMA method uses a simple arithmetic mean to estimate central tendency (UNWTO). Moving average provides a simple method for smoothing the "past history of data". The idea behind moving average is that observations which are nearby in time are also likely to be close in value. (Makridakisetal, 1998) The model allows the past values of a variable to determine the forecast values with equal weights assigned to the former. The number of lagged observations included in the model determines its responsiveness. The more included are the lagged values, the smoother the forecasts become. If a time series shows wide variations around a trend, including more lagged observations in the SMA model will help pick up the trend. However, its main limitation is that it gives equal weight to the demand in each of the most recent periods; it will not provide good forecast if data has a trend and a seasonal component (Buffa\&Sarin, 2011). This may not be realistic, as more recent lagged values tend to have a much bigger impact on the current values of a time series. Therefore, the SMA method normally generates more accurate forecasts where the time series is less volatile.

\section{Intermediate Extrapolation methods Single Exponential smoothing (SES)}

The single exponential smoothing model is applicable when there is no trend or seasonality in the data (Buffa\&Sarin, 2011). However, when the trend and seasonality component is absent what remains is the horizontal component or alternatively, the base. Therefore, the key objective in the exponential smoothing model is to estimate the base and use that estimate for casting future demand (Buffa\&Sarin, 2011). In the SES model, the forecast for period $\mathbf{t}$ is equal to the forecast for period (t-1) plus a smoothing constant multiplied by the forecasting error incurred in period (t-1). This is a process of determining 
Forecasting Tourist Arrivals and Supply and Demand Gap Analysis

by taking forecast for the previous period and adjusts it using the forecast error. "The new forecast is simply the old forecast plus an adjustment for the error that occurred in the last forecast. Here, the error of the past forecast is used to correct the next forecast in the direction opposite to that of the error" (Makridakisetal, 1998).

\section{Double exponential smoothing (Brown's DES)}

A number of models have been proposed for double exponential smoothing.However, the simplest one was Brown's DES. Brown's model was developed to deal with time series that has a linear trend over time (Fretchling D.C, 2001). If there is no trend, it reduces to the SES (Peng Bo, 2012). The advantage of DES is that it can capture linear trends up or down and forecast several periods ahead. However, it cannot track non-linear trends. It fails to simulate stepped series well and cannot deal effectively with seasonality (Fretchling D.C, 2001),(UNWTO \& ETC, 2008).

When it comes to forecasting tourist arrivals, simple and intermediate extrapolation methods have their own drawbacks/limitations. They are commonly used for short term forecasting; when there is no trend and no seasonality, they are used as a control method when assessing whether other forecasting methods are worth using (Buffa\&Sarin, 2011), (UNWTO \& ETC, 2008), (Fretchling D.C, 2001). In light of the limitation on simple (basic) as well as intermediate extrapolative methods, the circumstances lead to the use of an advanced extrapolative method i.e. the Box-Jenkins or Causal approach. However, since the main interest here is to select a model for tourism data analysis, the data type is a univariant time series. The appropriate method to be used here is the Box-Jenkins approach. The Box-Jenkins approach by itself is a combination of two forecasting methods which are Auto Regression and Moving Average. Time series models have been widely used for tourism demand forecasting in the past four decades with the predominance of the integrated autoregressive moving-average models (ARIMAs) proposed by Box and Jenkins (1970). Different versions of the ARIMA models have been applied in over Sixty percent of the post-2000 studies that utilized the time series forecasting techniques (Song \& Li, 2010).

\subsection{Application Software for Box-Jenkins Methods}

Standard computer packages like SAS, SPSS, TRAMO-SEATS (TS), X-12ARIMA, and statistical packages are available for Box-Jenkins method. The computer package used in this analysis is X-12-ARIMA which is the seasonal adjustment package. It has been one of the leading statistical packages 
Forecasting Tourist Arrivals and Supply and Demand Gap Analysis

available for seasonal adjustment methods for use in official statistics in many countries: United States, Canada, and Europe to state a few. The method is used by most of the leading national statistical institutes, national banks, academics and researchers across the world. X-12-ARIMA is developed by the United States (US) Bureau of the Census. The software is comprehensive, with many options available for tailoring seasonal adjustments to each individual series. It is made freely available by the US Bureau of the Census.

\section{Research Methodology}

Under this section, data presentation, preliminary analysis, model identification and selection, methodology is used and output analysis is presented.

\subsection{Data Source Presentation and Analysis}

Data Source: The data source for this analysis is the Ministry of Culture and Tourism (MOCT), Policy, Planning, Evaluation and Monitoring Directorate Publication, Tenth Tourism Statistics Bulletin 2009-2012 (MOCT, 2013). The data used is Arrival of Tourists at the National Border by Month, from 2006 up to 2012 .

Source data presentation: the plot of the monthly tourist arrival (MTA) original series is presented here.

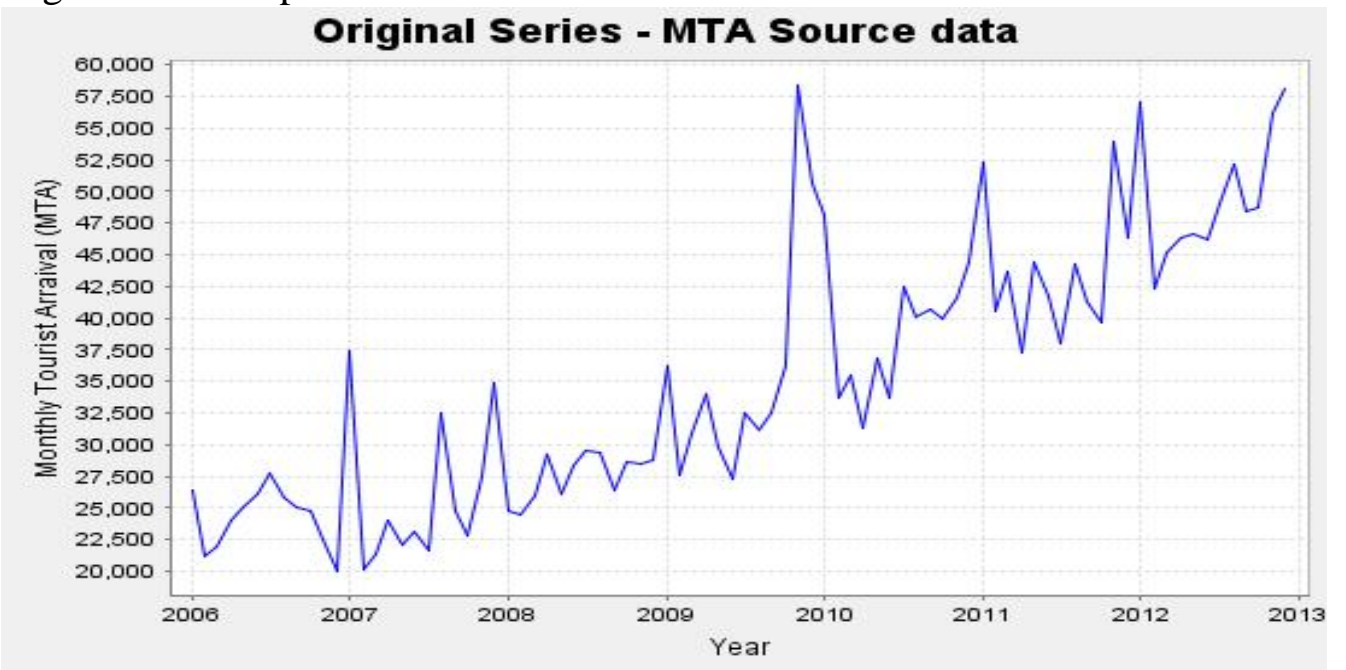

Fig 3.1: Original data series plot

The plot of the monthly tourist arrival (MTA) reveals important information about the data characteristics. There are periodic picks which are observed from time to time. The picks are observed at and around the beginning of each 
year followed by a low season. Although it is not as big as the beginning, there is also a rise in pick before and after midyear periods. This indicates that there are periods which have a higher arrival rate than other periods within the year which, in turn, gives an indication for the seasonal nature of the observation. The plot also shows a positively increasing behavior with time. This signifies the presence of a linear trend component or an increasing mean for the series. On the other hand, it is difficult to pinpoint the observed characteristics of the amplitude of the variation. It is not that clear if the series has increasing seasonal amplitude or it is relatively a constant one. But it is clear that the amplitude variation did not show consistent behavior. It is more of an erratic behavior where as it shows a reasonable and similar periodic repeating pattern with an increasing trending component.

\subsection{Model Identification and Selection}

The first step in the analysis of the Box-Jenkins method is the use of the model identification procedure. The procedure involves plotting of the initial data. The source data is run to determine the appropriate model. The Box-Jenkins procedure requires the data to be stationary. This requires input data to be adjusted to form a stationary series, one whose value may vary more or less uniformly about the fixed level. But before directly going to this step, one needs the answer to this question, "Does the data require being made stationary?" This can be answered by analyzing the plot of the data.The ACF and PACF plot of the series can give indications of whether it is necessary to change or transform the data into a stationary series. If the initial data series display neither trend nor seasonality and residual plot shows values within the confidence interval. Then the process can be continued without any hindrance. Sample Autocorrelations of the Series Differencing: none 
Forecasting Tourist Arrivals and Supply and Demand Gap Analysis

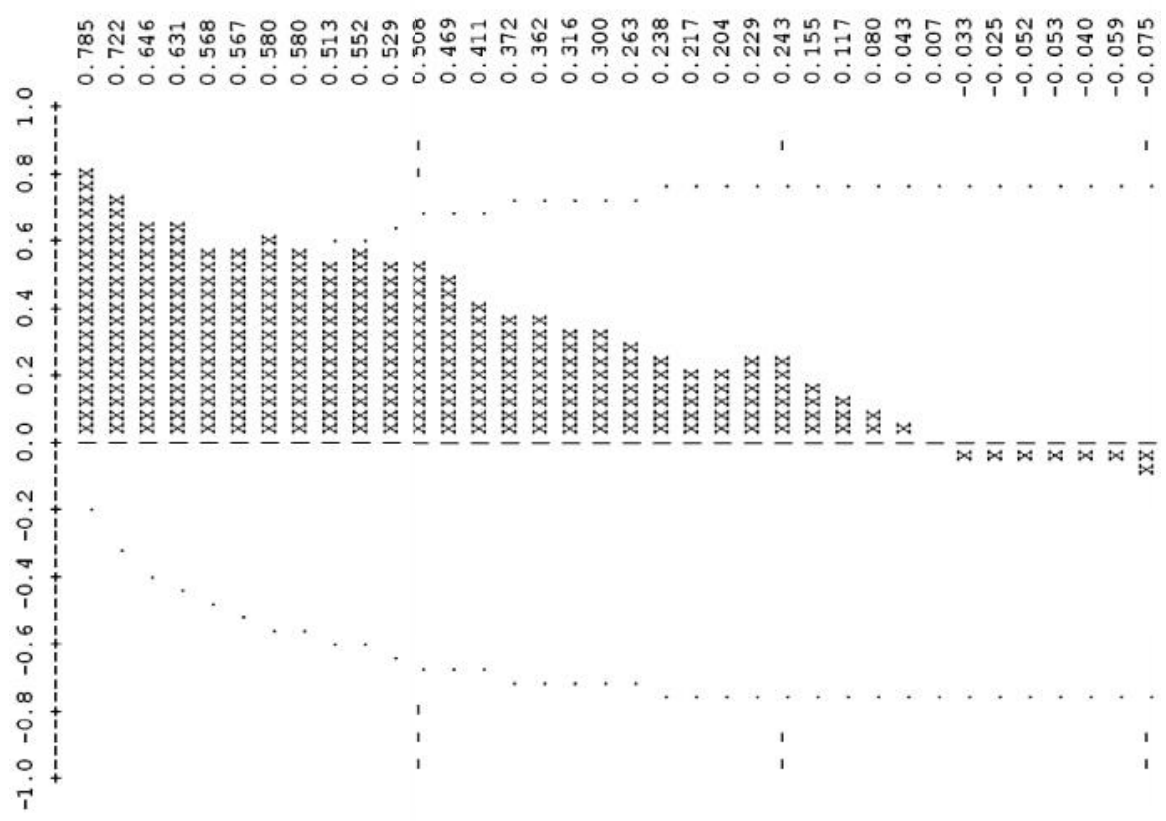

$\rightarrow$ 때

Fig 3.2: Sample auto correlation of the series: No differencing

Sample Partial Autocorrelations of the Series Differencing: none

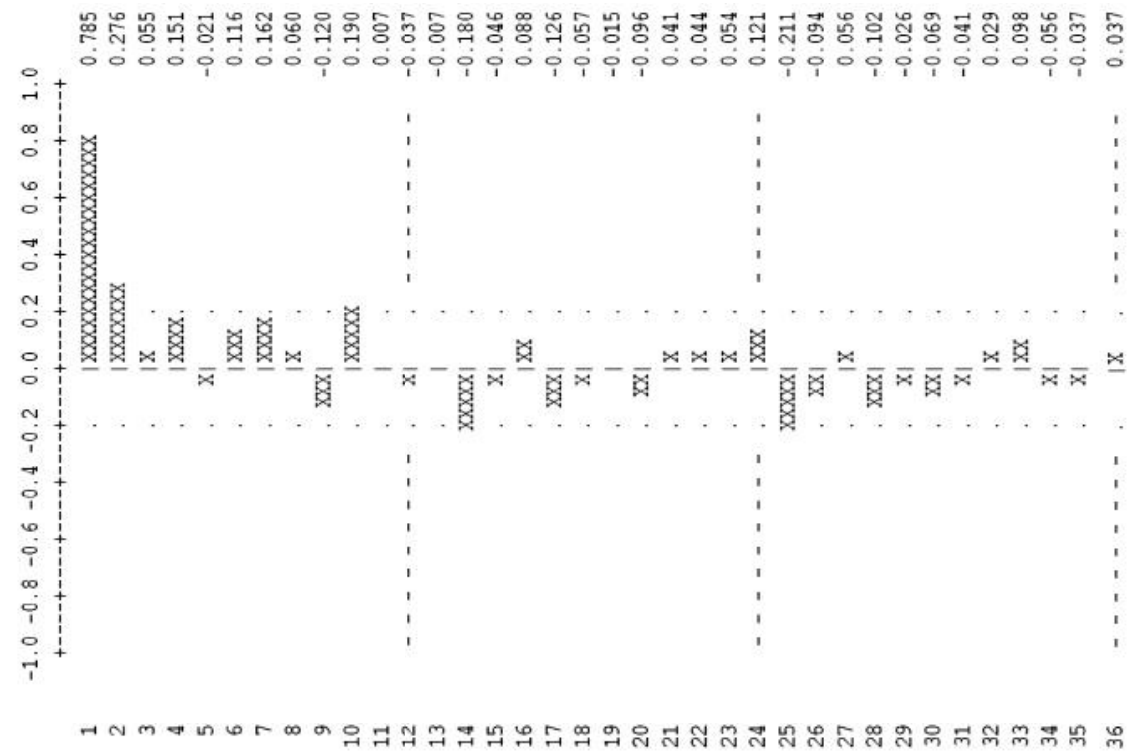

Fig 3.3: Sample partial Auto correlation of the series: No differencing

EJBE Vol. 5 No. 2/2015

Page 282 
Forecasting Tourist Arrivals and Supply and Demand Gap Analysis

The Auto Correlation Function (ACF) of the series shows a gradual decrease (decay) towards zero. The plot shows significance up to lag 8, although it is not much pronounced due to weaker signals, there is an indication that the gradual decrease shows picks at seasonal lags around lag 12, 10, 11,12, 24.

The Partial Auto Correlation Function (PACF) plot shows that there is visible significance at lag 1 and 2 while the rest is within the range of boundaries for the white noise criteria or not significant from zero. The significance on lag one (1) and lag two (2) may suggest the preferred model to be AR (2).

Over all, the plot of the series has provided information about the characteristics of the series. The series has an increasing trend, show fluctuation in periods within a year which indicates seasonality, the ACF shows significance up to lag 8 and attenuates gradually towards zero. Therefore, it is possible to conclude that the series is not stationary;as a result, it needs to be differenced and/or transformed.

\subsection{Models Selection}

Model selection process starts with taking advantage of the auto selection feature of X-12-ARIMA. The program is allowed to run on Auto mode; it uses the log transformation and selects ARIMA (311) (011). The plot for (311) (011) shows significance ACF at lag 4, 5 and 8. PACF shows significance at 4, 5 and 12.All the $\mathbf{p}$ values show a value less than 0.05 which indicates the poor fit of the model to the data. Therefore, the selected model cannot be considered.

From the pattern of behavioral investigation of the series for stationarity, it has been observed that the contribution of the log transformation in making the series stationary is not that visible while the seasonal differencing is critical. On the other hand, the auto model selection uses log transformation. In order to recheck the auto model selection option, the series is run by removing the log transformation effect. That means auto model selection using differencing only. However, results show the model selected is the same model (311) (011). It also shows similar $\mathbf{p}$ values which are less than 0.05 ; therefore, it cannot be considered as a suitable model. This prompts to check the manual model selection procedure.

In order to choose the most appropriate model, the manual selection process is used to generate an additional model option which will be compared with each other to find the best model to be used for forecasting. The manual selection process followed a structured system of investigation based on the principle of parsimony. Each model is checked for fulfilling diagnostic requirements. The

EJBE Vol. 5 No. 2/2015

Page 283 
models considered are based upon the observation of the series' characteristics which exhibits strong seasonal component (011) from the earlier test.

MODEL 1 - SARIMA (110) (011) 12

The result for model (110) (011) shows no significance ACF, however, its diagnostic shows LBQ fail at lag 2, significance LBQ 3-4, significance $\mathrm{BPQ}=3-4$, significant $\mathrm{PACF}$ 2, $\mathrm{AIC}=1392.3906, \mathrm{AICC}=1393.7031, \mathrm{P}$ Value $<$ 0.05 or $5 \%$ at lag 1 and lag 2 , as a result, the model is rejected based on the diagnostic result.

MODEL 2 - SARIMA (211) (011) 12

The plot of the series shows no significant ACF and PACF. All p values are greater than 0.05 or $5 \%$, AIC Value $=1385.8193$, AICC Value $=1388.1419$. This model qualified for consideration.

Model 3 - SARIMA (111) $(011)_{12}$

The plot of the series shows no significant ACF and PACF. All p values are greater than 0.05 or $5 \%$, AIC Value $=1383.8612$, AICC Value $=1385.6389$. Model is qualified for consideration.

Model 4- SARIMA (011) (011) 12

The plot of the series shows no significant ACF and PACF. All p values are greater than 0.05 or $5 \%$, AIC VALUE $=1381.9061$, AICC VALUE $=1383.2186$. Model is qualified for consideration

MODEL 5 -SARIMA (210) (011) 12

Diagnostic show LBQ and BPQ FAIL at lag 4 , P value at lag $4=0.041$ which is less than 0.05 or $5 \%$, AIC VALUE $=1387.3385$, AICC Value $=1389.1162$. Model rejected based on diagnostic result.

Model 6- SARIMA (312) (011) 12

The plot of the series shows no significant ACF and PACF. All p values are greater than 0.05 or $5 \%$, AIC Value $=1385.8193$, AICC Value $=1388.1419$, AAFCE $(3 y r)=6.648$. Model is qualified for consideration.

Model 7 -SARIMA (212) $(011)_{12}$

The plot of the series shows no significant ACF and PACF, all $\mathbf{p}$ values are greater than 0.05 or $5 \%$.for all df $>0$, AIC Value $=1387.3709$, AICC value $=1390.3217$. Model is qualified for consideration.

MODEL 8 -SARIMA (112) (011) 12

The plot shows no significant ACF but it has significant PACF at lag 5, diagnostic show $2 \mathrm{LBQ}$ fail at lag 5 and 6 significant $\mathrm{BPQ}=1$ at lag $5, \mathrm{P}$ value at lag $5=0.015$, p values at lag $6=0.046$ which are less than the threshold value of 0.05 or $5 \%$, AIC Value $=1395.0968$, AICC Value $=1396.8746$. Model is rejected based on diagnostic result.

Model 9-SARIMA (012) (011) 12 
Forecasting Tourist Arrivals and Supply and Demand Gap Analysis

The plot of the series show no significant ACF and PACF. All $\mathbf{p}$ values are greater than 0.05 or $5 \%$, AIC Value $=1383.8648$, AICC Value $=1385.6425$. Model is qualified for consideration.

The list of qualified models for consideration is: (211) (011), (111) (011), (011) (011), (312) (011), (212) (011), and (012)(011).

\begin{tabular}{|c|c|c|c|c|}
\hline No. & $\begin{array}{c}\text { Model of } \\
\text { forecasting } \\
\text { (X-12- } \\
\text { ARIMA) }\end{array}$ & AIC & AICC & $\begin{array}{c}\text { Average absolute } \\
\text { percentage \% Error } \\
\text { (AAPE, last 3 years) }\end{array}$ \\
\hline 1 & $(211)(011)$ & 1385.8193 & 1388.1419 & 6.56 \\
\hline 2 & $(111)(011)$ & 1383.8612 & 1385.6389 & 6.52 \\
\hline 3 & $(011)(011)$ & 1381.9061 & 1383.2186 & 6.02 \\
\hline 4 & $(312)(011)$ & 1389.3701 & 1393.0368 & 6.64 \\
\hline 5 & $(212)(011)$ & 1387.3709 & 1390.3217 & 6.58 \\
\hline 6 & $(012)(011)$ & 1383.8648 & 1385.6425 & 5.98 \\
\hline
\end{tabular}

Table 3.1: Qualified ARIMA Models

From the tabulation of the selection parameters, there are competing models which can satisfy the combined parameters of lowest AIC and AAPE.However, the model which fulfills the selection criteria with the lowest AIC value of 1381.9061 among qualified models is (011) (011); it has the lowest AIC and AICC value but has slightly higher AAPE which is 6.02. Model (012) (011) has the lowest AAPE of 5.98 for the last 3 years but its AIC is 1383.8646 which is higher than model (011) (011). However, the difference in AAPE values between the models (011) (011) and (012) (011) are not that significant. It only shows a marginal difference of 0.04 which gives a winning edge for model (011) (011). The rest of the models (211) (011), (111) (011), (312) (011), and (212)(011) have a higher AIC value than the main front runner models, therefore, they are dropped. As a result, model (011) (011) is selected to be used as the model for the MTA series. For this reason, it is used in the forecasting process. 
Forecasting Tourist Arrivals and Supply and Demand Gap Analysis

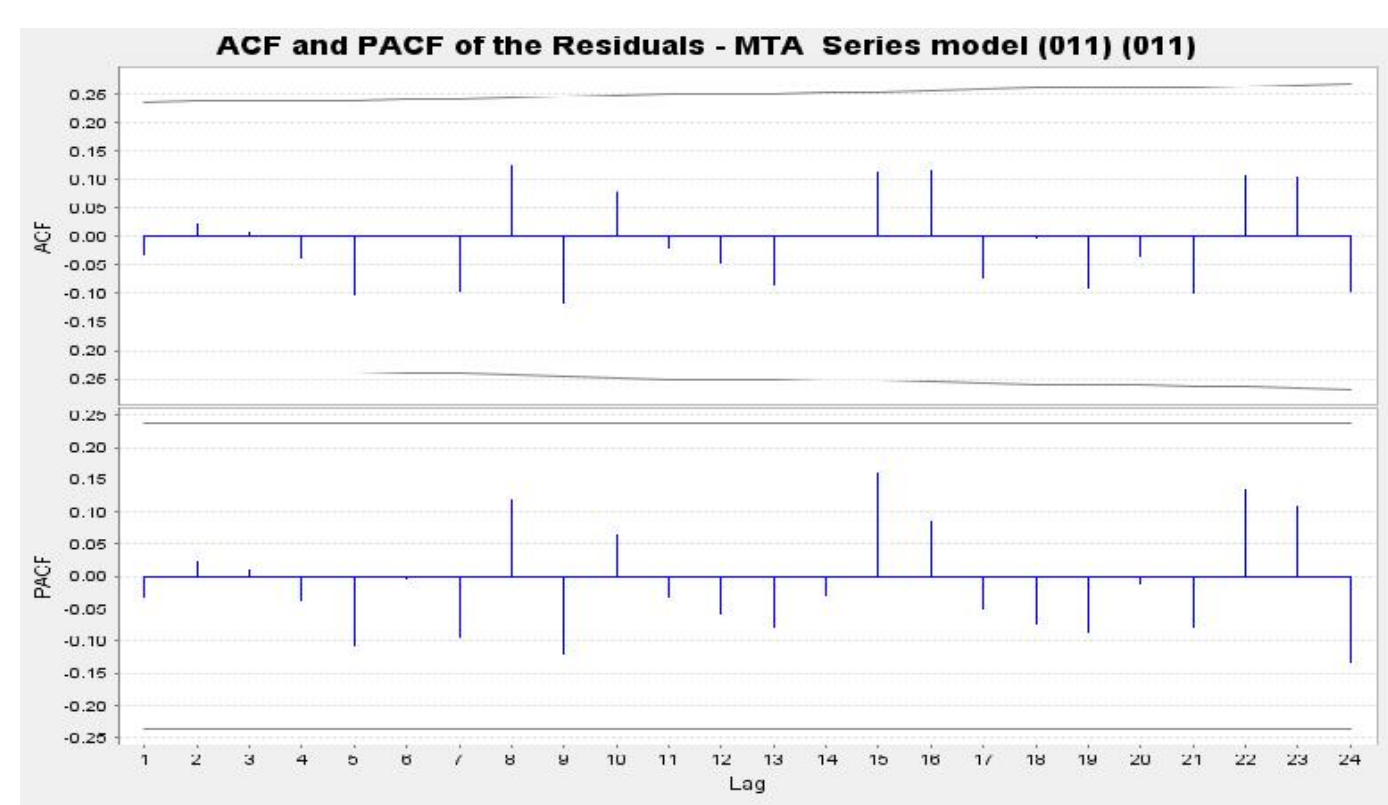

Fig 3.4: ACF and PACF of the residual; Model (011) (011) 12

\subsection{Supply and Demand Gap}

The methodology used to determine the supply and demand gap is the funneling technique; it basically works by decomposing or breaking down the bulk arrival figure into its different elements. It starts with the lump sum arrival figures, identifying the city component from the overall arrival, and then it further looks into the family and friends visitor element from the city visitors, as this category of visitors are mostly composed of people who have family and friends. They are not expected to look for accommodation facilities in the city hotel establishments, however, some portion of this category of visitors is considered to use accommodation facilities. This provides the number of visitors who are looking for accommodation facilities. The final filtering comes in with identifying the actual room counts required on daily bases which also requires considering the possible double occupancy factor.

The process enables us to convert the total forecast of annual tourist arrivals of the country to visitors who will stay and look for accommodation facilities in the city. It becomes the basis of the demand for hotel or accommodation rooms for the city of Addis Ababa. 
Forecasting Tourist Arrivals and Supply and Demand Gap Analysis

\subsection{Scenario Planning and Intervention \\ 3.5.1 Intervention}

When a time series data is impacted by extreme change, (the change could be positive or negative and it could come from external or internal factors) it affects the mean of the series. Such change is called change due to intervention effect. It is also called structural change or regime change (Z Ismail, 2009). Change from external factors could be manmade or natural. For instance, external factors could emanate from weather factors, volcanoes, earth quakes, tsunamis, and diseases (Sars, Foot \& Mouth, and Ebola). Alternatively, it could be manmade interventions like embargos, political/policy change, and terrorism; it could also be due tovarious economic factors like the effect of economic recessions, the increase in economic growth, disposable income and interventions like policy impact and increased promotion of destinations.

The general procedure for an intervention analysis is mostly post impact analysis which divides the period before and after. Whereas in this case, what is attempted is the prediction of the impact an intervention has before it happens; this is done to see the case of a different scenario.

\subsubsection{Scenario Planning}

This is an attempt to visualize a number of possible futures and consider their implications. A major purpose of scenarios is to challenge conventional thinking and avoid extrapolating into the future in a linear fashion (Makridakisetal, 1998). It works by constructing possible alternative futures, or scenarios. Accordingly, scenario planning coupled with time series intervention effect is used to generate the possible alternative scenarios of long term forecasting of tourist arrivals as it is indicated on the graphs. This part of the process complements the quantitative analysis with the judgmental (qualitative aspect) to generate alternative options.

\subsection{Output Analysis}

\subsubsection{Output Report}

The output of the program using the selected model has produced many tables. In fact, the X-12-ARIMA program has extensive list of outputs; it is not practical to address all the reports, therefore selected reports are presented. 
Forecasting Tourist Arrivals and Supply and Demand Gap Analysis

\subsubsection{Presenting Forecasted Series}

The selected model (011) (011) 12 is used to generate long periods of forecast by re-plugging the result of earlier forecasts as input data for forecasting the unknown future values. This method has created a way to extend the forecasts to distant periods up to year of 2025 which in turn presents the opportunity to get long term forecasts of monthly tourist arrivals (MTA).On the other hand, the Quarterly Tourist Arrivals (QTA) or the quarterly series, made available by following similar rigorous procedures as MTA, has been followed and presented to complement MTA. The model used for QTA is (210) (011) 12 . However, it is important to note that there is a challenge in quarterly forecasts when it comes to extending the forecast period beyond 2020 .

In general, it is also important to provide a word of caution about attempting to extend the forecast period. The model cannot be effective when extending the time period indefinitely. One of the main issues of model is that it might not meet diagnostic requirements. Even a new model might not succeed in complementing the data properly (experience of the quarterly series shows this).On the other hand, when one wants to explore the distant future, there is no guaranty that conditions will remain the same; there is always a possibility that exogenous factors can change and influence the dependent parameter.

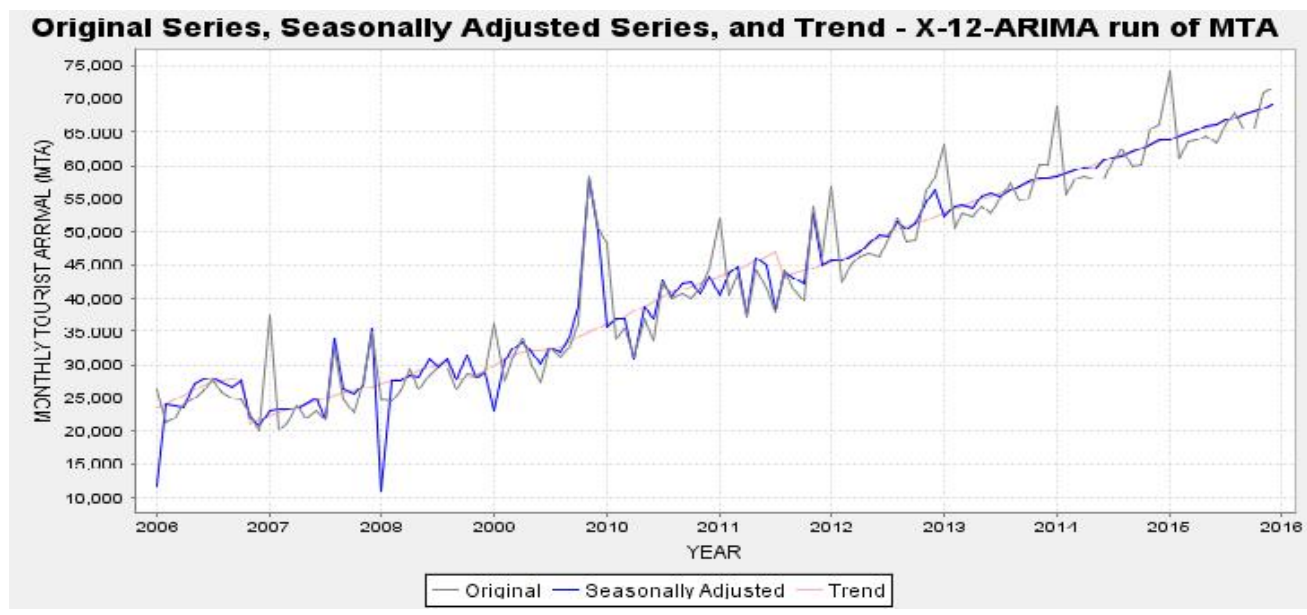

Fig 3.5: X-12-ARIMA Run of MTA source data 
Forecasting Tourist Arrivals and Supply and Demand Gap Analysis

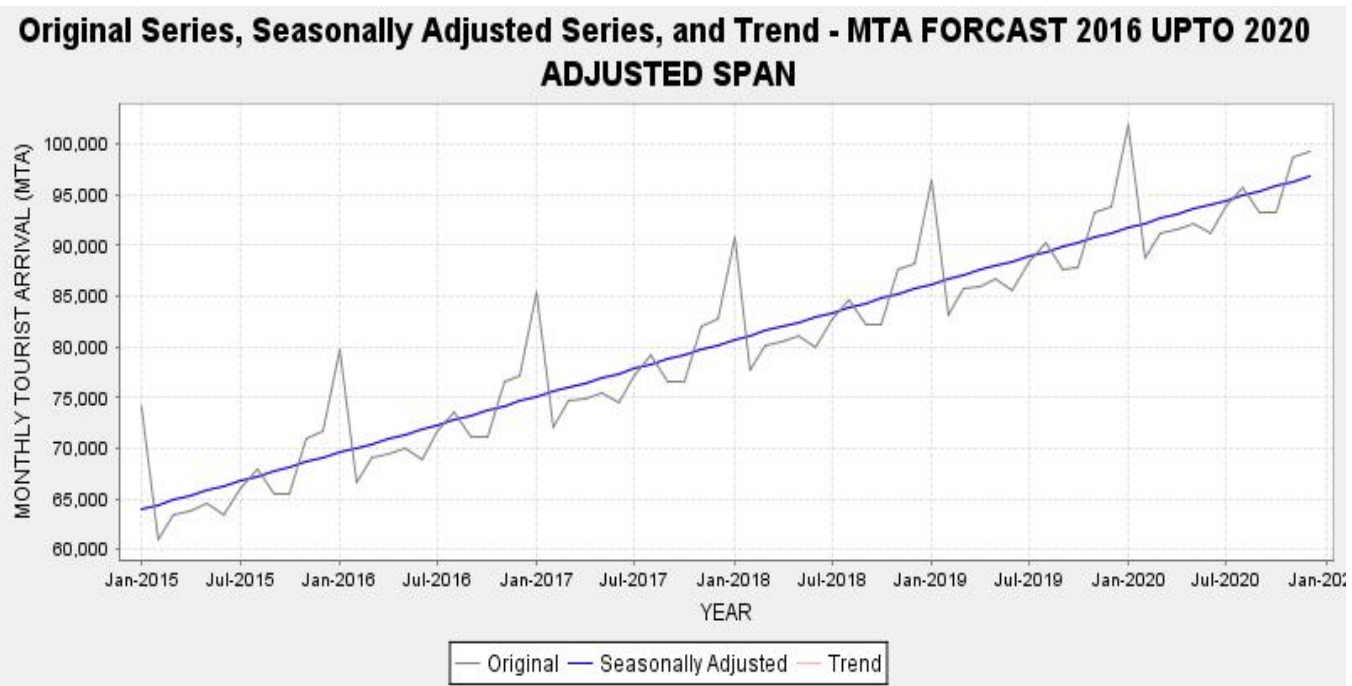

Fig 3.6: MTA Forecast, 2016 up to 2020

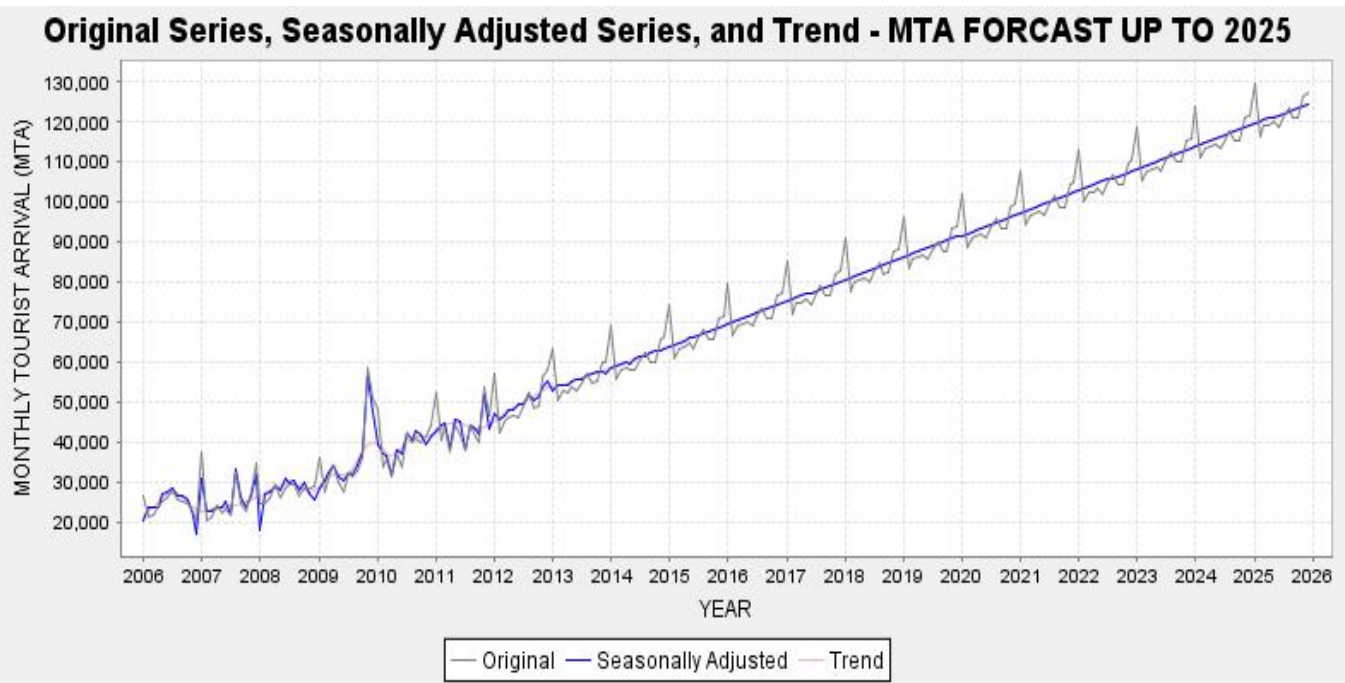

Fig 3.7: MTA Forecast up to 2025 
Forecasting Tourist Arrivals and Supply and Demand Gap Analysis

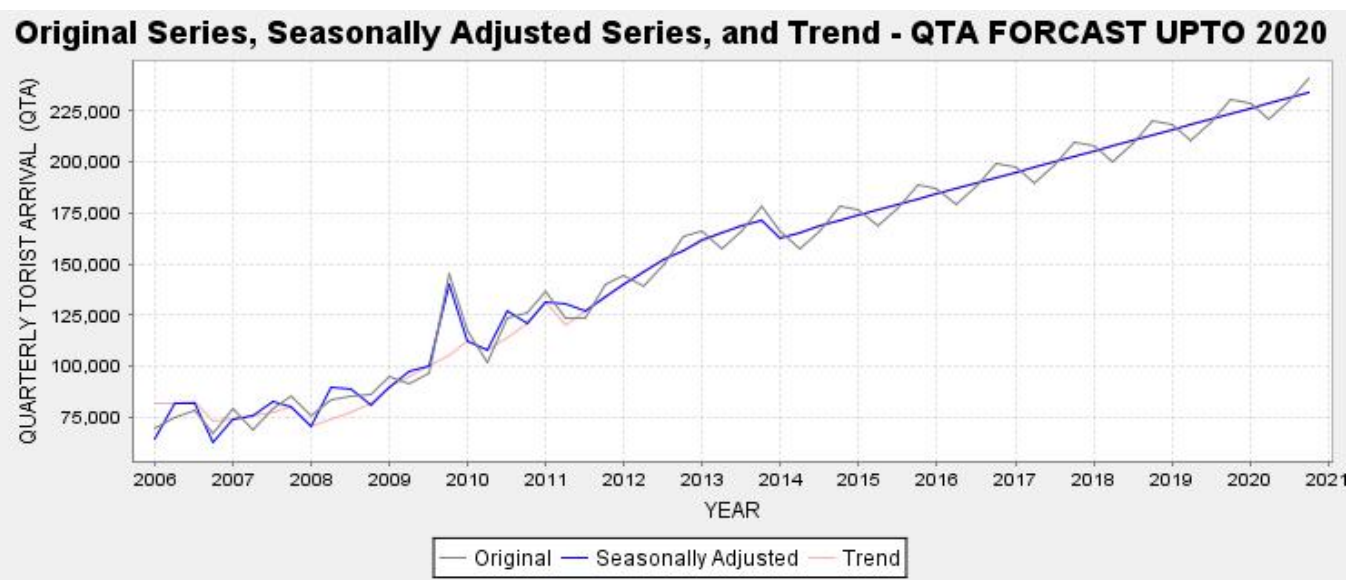

Fig 3.8: QTA Forecast up to 2020

\subsubsection{Interpretation of Output}

Original Series: this series of the output shows the typical randomness of the arrival pattern up to end of the actual observed data (2006-2013). The plot exhibits similar repeating patterns. As of 2013 which is the starting point of the forecasting period and extends up to the year of 2025. The repeating pattern is clearly indicated on the adjusted span plot.

Seasonally Adjusted Series:the aim of preparing seasonally adjusted series is to adjust or correct the seasonal effect which masks the underlying trend of the series. The correction is intended to reduce the variability induced by the seasonal component of the data. Therefore, it is natural to exhibit the characteristics of the original series with more smoother or subdued variations. The plot also shows this fact as well as showing the randomness which follows the original series, though it misses certain points of picks and trough. In the forecasted period as of 2013, the seasonally adjusted series shows more smooth lines or linear characteristics this is so because the model representation for the original series has less randomness or variability in this period.

Trend: the trend plot of the series exhibits more smooth linear patterns. This results from the characteristics of the underlying growth pattern and trend of the series over time. The pattern shows abrupt level changes or shifts in level in the mid-year of 2011. This change is also observed in the original as well as on the seasonally adjusted series. The trend line coincides with the seasonally adjusted series in the forecasted period up to the end of 2025 .

EJBE Vol. 5 No. 2/2015 
Forecasting Tourist Arrivals and Supply and Demand Gap Analysis

\subsubsection{Seasonality Analysis}

Seasonality is a notable characteristic of tourism demand and cannot be ignored in the modeling process when monthly or quarterly data are used (H.Song\&G.Li). Accordingly, seasonality has been observed on the modeling of the monthly tourist arrival (MTA). In his case, although the seasonality is there, its manifestation is not that strong. The non-parametric test of the model shows the presence of seasonality at $1 \%$ and moving seasonality is presented at $5 \%$.

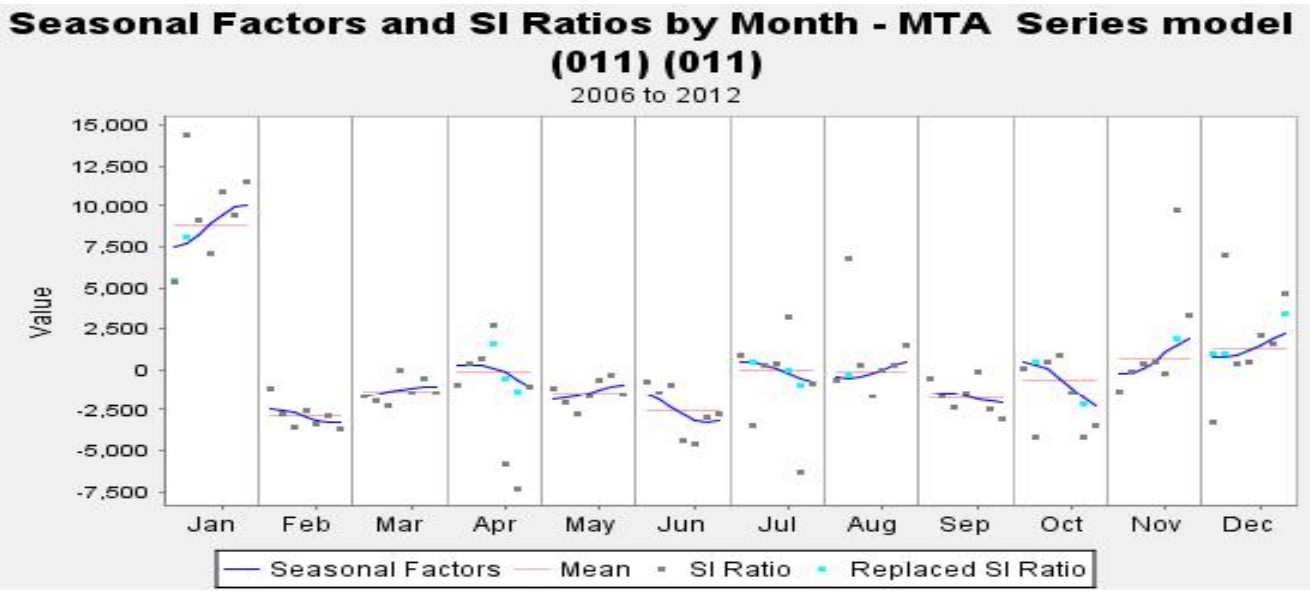

D-10 plot of the result. (Seasonal factor)

Fig 3.9: Seasonal Factors and SI Ratio: MTA series model (011) (011) 12

The seasonal factor plot shows a positive trend on November, December and January; while January exhibits the pick of the season by a factor of 8836 . On the other hand, February exhibits the opposite or trough with a factor of -2898 . March and April show recovery from February and they exhibit factors of 1297 and -113 , whereas May and June show factors of -1434 and -2593 respectively. June exhibits the second lowest factor. However, the dip is not as big as February. April exhibit an identifiable recovery. Again, July and August exhibit identifiable positive recoveries which are -21 and -136 . Whereas September and October exhibit low factors of -1702 and -677 which show reduction from arrival levels of July. It could possibly be related to the low period of movement (spring) in tourist generating countries. The pick in January could probably be attributed to increase in visitor interests to experience the Ethiopian Orthodox Church calibrations of Christmas at Lalibela and Timket (Epiphany) in Gonder. The survey result published by MOCT in 2013 supports this argument as it shows $42.89 \%$ of visitors are 
Forecasting Tourist Arrivals and Supply and Demand Gap Analysis

coming to experience the cultural attractions, whereas $27.73 \%$ of visitors are interested in historical attractions. The increased number of visitors in November and December could be attributed to the convenient weather factor in the country at that time. However, despite the same convenient weather factor, the trough in February is something which requires further investigation. Despite Ethiopia's rainy season, the relative arrival increase in July and August could probably be attributed to Tourist Season (summer vacation) in tourist generating countries, specifically Europe and USA. The same pattern of the monthly variation in arrival (seasonality) is observed in the forecasted period ;however, the value has increased. For example, January has more positive pickups whereas February shows a relative reduction in the level of the trough.

\subsection{Trend Analysis}

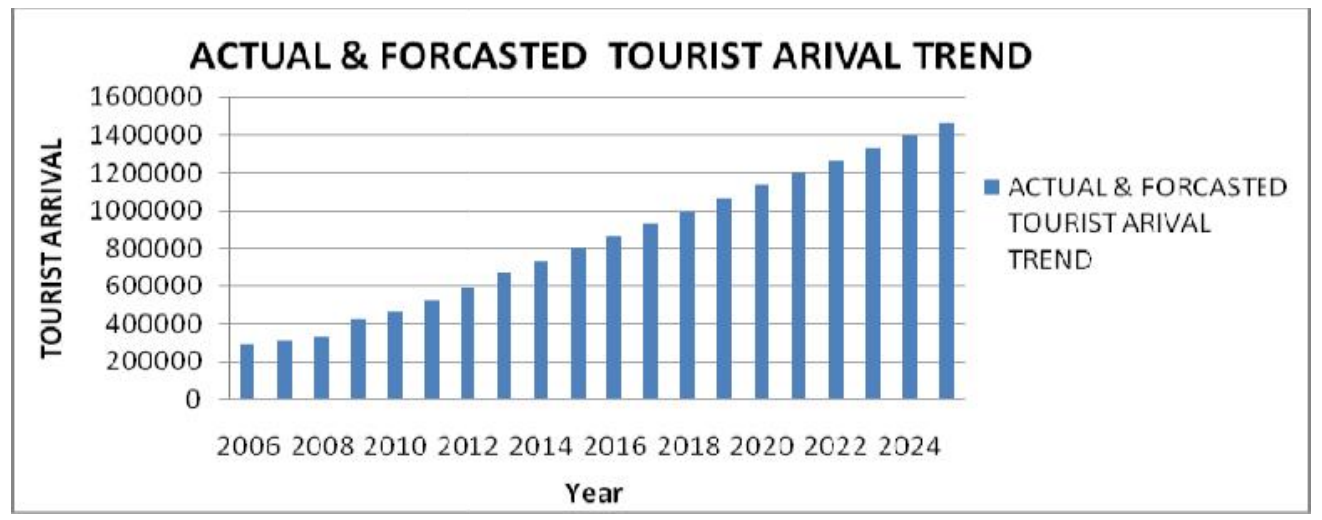

Fig 3.10: Actual and Forecasted tourist arrival trend

The trend analysis shows that the annual average growth between 2006 and 2025 is $9 \%$. Whereas the annual growth between the actual observation period of 2006 to 2012 was $13 \%$. The biggest annual increment or growth in arrivals is observed between 2008 and 2009 which was $29.42 \%$. The minimum growth is observed at the end of the forecast period with $4.76 \%$ annual growth rate. The annual growth trend shows gradual decrease when the forecast period goes further and further out. The decline in growth rate starts at the end of actual observation period (2011/2012) which had an annual increment of $13.92 \%$. The growth gradually decreases to annual increments of $4.76 \%$ at the end of forecast period 2025. On the other hand, the monthly mean for the forecast period comes to 97,021 ; the minimum monthly arrival is 69,590 which mostly happen in the month of February, whereas the maximum monthly arrival 
Forecasting Tourist Arrivals and Supply and Demand Gap Analysis

comes 124,520 in the month of January. The standard deviation comes to 16,009 .

\subsection{Supply and Demand Gap}

To further explain/highlight the methodology used for the supply and demand analysis, the forecast figure for the years ahead is used as initial input (lump sum arrival) figure which is then used on the funneling technique. It is decomposed until it reaches the final and filtered actual room demand. The process enables us to convert the total forecast of annual tourist arrivals of the country to visitors who will stay and look for accommodation facilities in the city. This will become the basis for the hotel or accommodation demand in the city.

\subsubsection{Intervention}

The type of intervention considered here is the stepped function which causes permanent level changes. For further clarification on the stepped function, refer to the works of Yafee \& McGree (2009). The impact is expected to manifest within a year, in this case, 2016.The model used is the same model before and after the impact.

\subsubsection{Consideration of Different Scenarios}

Tourism is a delicate sector which can be easily impacted by exogenous factors. Although it is very challenging to predict what is going to happen, from past experiences, it has been observed that exogenous factors have considerable influence on tourist arrivals. Such an influence can come at any time when one deals with long term forecast. Accordingly, the impact of exogenous factors in 2014 has affected tourist arrivals in East and South African countries like Kenya, Tanzania and South Africa; the observations from these countries have been taken as a learning point. The official release from these countries' tourism biro, hotel chains, and tour and travel companies has shown that the Ebola pandemic in West Africa has affected their tourist arrivals heavily; in the case of Kenya, this is more aggravated by security concerns from Somali extremist groups,,especially in tourist destinations like Mombasa. For example, the Hotel Association of Tanzania representing 195 nationwide sites claimed that business is down by 30 to 40 percent for the year and advanced bookings, mostly for 2015, are 50\% lower. Kenya's Serina Hotels which are high end safari lodges and beach resorts announced booking is down as much as 30\% in 2014. Leisure Tourism(2014), a Netherland based 
Forecasting Tourist Arrivals and Supply and Demand Gap Analysis

safari booking has conducted a survey on more than five hundred operators and announced booking is down by 20 to $70 \%$. South African tour operators and tourism officials have also confirmed the drop on their bookings especially from Asian travelers. The World Bank also cut the forecast of economic growth for sub Saharan Africa from 5.2 to $4.6 \%$ (Leisure Tourism, October 2014). Considering the implication of such external factors and the sensitivity of the tourism industry, the reduction of $15 \%$ from normal trend forecast is taken as the case for worst case scenario.On the same premises, a positive impact of 15\% increase in tourist arrival is also assumed (Note that the standard deviation to mean arrival ratio show 16.5\%). On the other hand, considering the Ethiopian government's intention to prop up support for the industry in the coming years, the third case scenario considers a stretched goal.The Ethiopian government's GTP plan has been aimed to reach more than a million tourist arrivals by 2015. However, the current trends show that it is highly unlikely that the target will be met. The forecast of this analysis also shows that with the current trend it will be challenging to meet the target without some major intervention in the upcoming years. Therefore, this scenario is a bit of a stretch considering that it takes the current trend forecasted figure for 2025 to be taken as the target to be achieved by 2020 . Similar strategic goals are observed in East African countries' (Kenya, Tanzania, Uganda, and Rwanda) tourism plans. This shows that it is possible to consider the achievement of such number of arrivals within the set time frame. The fourth case scenario is taken as the forecast of the current trend.

\section{Presenting Room Demand}

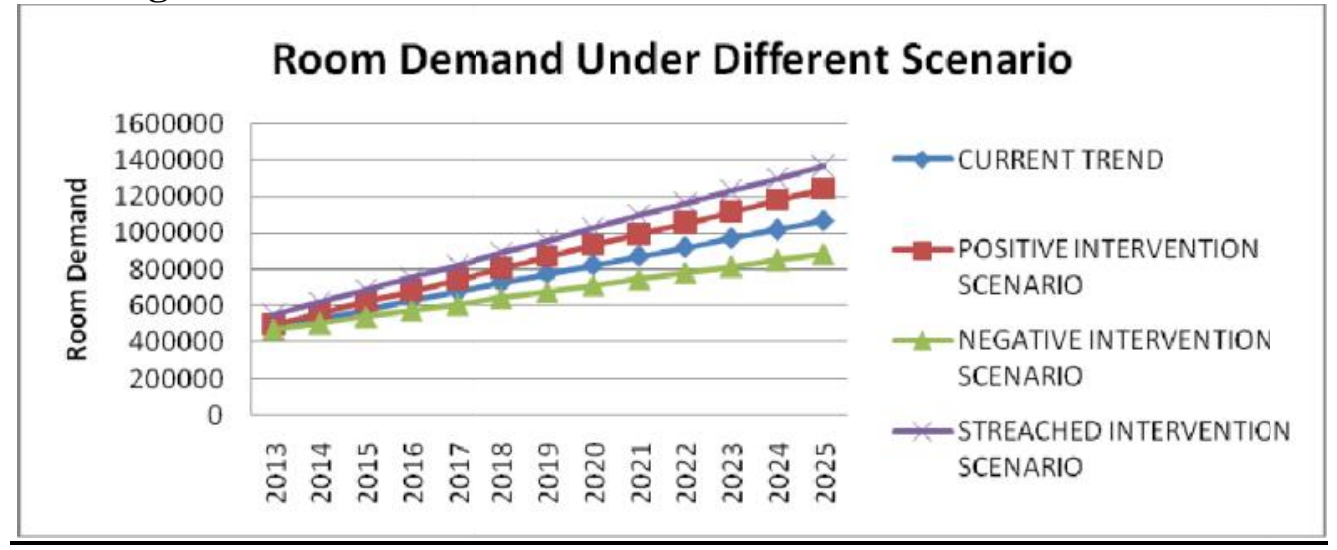

Fig 3.11: Room demand under different scenario 
Forecasting Tourist Arrivals and Supply and Demand Gap Analysis

\subsubsection{Establishing Supply Condition}

To establish the existing supply condition, it was important to visit stakeholders in tourism and hotel sectors. Accordingly, different government and sectorial associations (organizations) were approached. The main sources of information used are the Addis Ababa Tourism Office, operating under Trade and Industry Sector, Addis Ababa Investment Bureau,as well as Addis Ababa Hotel Owners Trade Sectorial Association (AHA).The information obtained from these different sources is examined to establish the current stock of hotel rooms.

The expected annual room supply condition can be indicated below.

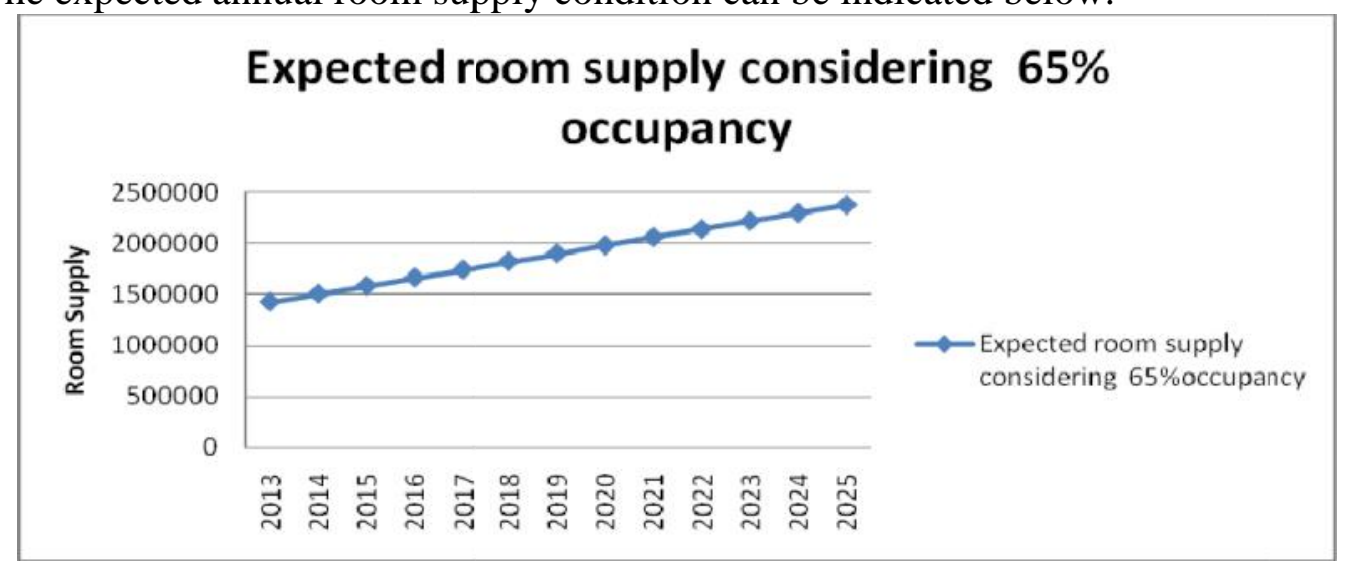

Figure 3.12: Expected room supply considering 65\% Occupancy 
Forecasting Tourist Arrivals and Supply and Demand Gap Analysis

\subsection{Gap Analysis}

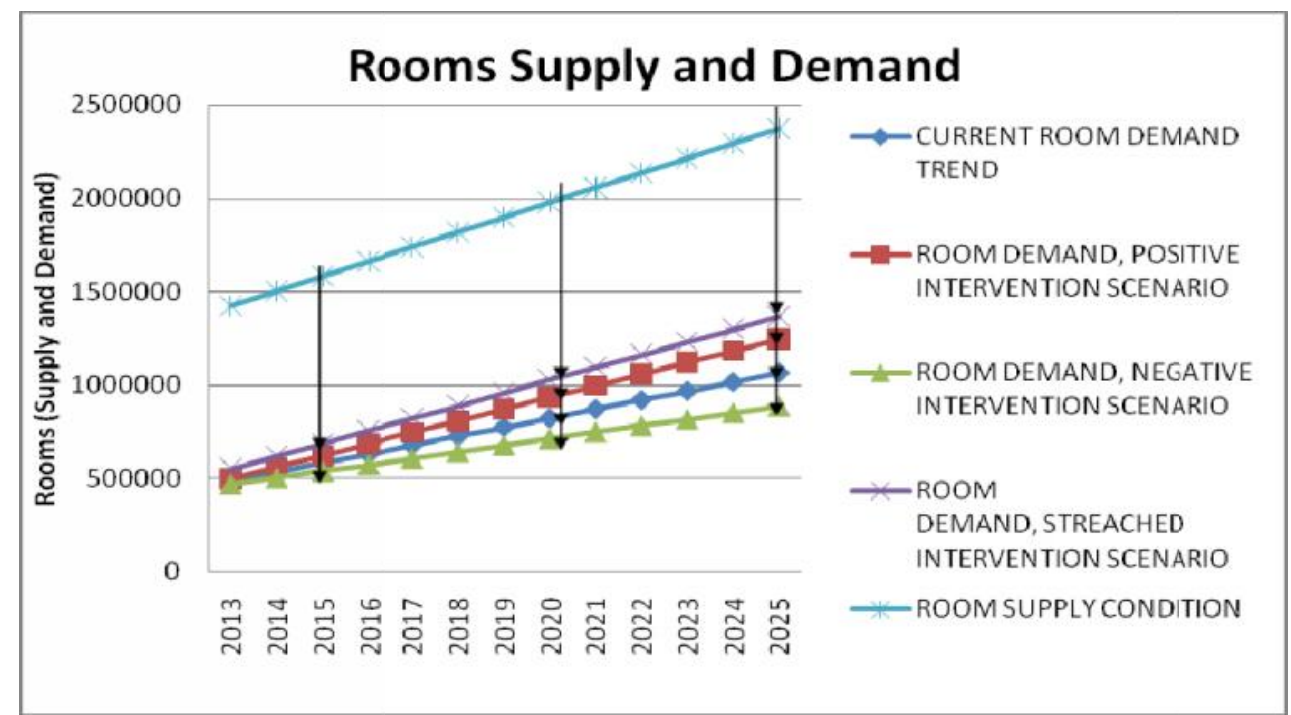

Fig 3.13: Gap analysis: Room supply and demand

The plot of the supply and demand indicates the gap between the supply (available rooms to be rented), presented on the upper side, in relation to the demand which is presented on the lower side for different scenarios. In all cases, the supply by far outstrips the demand.

Presenting the gap between supply and demand for different case scenarios:

\begin{tabular}{|l|c|c|c|}
\hline \multirow{2}{*}{$\begin{array}{l}\text { Supply vs. demand Different case } \\
\text { scenario }\end{array}$} & $\mathbf{2 0 1 5}$ & $\mathbf{2 0 2 0}$ & $\mathbf{2 0 2 5}$ \\
\cline { 2 - 4 } & 1000639 & 1154101 & 1307564 \\
\hline $\begin{array}{l}\text { Supply vs. demand gap (actual trend) } \\
\text { intervention scenario) }\end{array}$ & 1043291 & 1265532 & 1487772 \\
\hline $\begin{array}{l}\text { Supply vs. demand gap (positive } \\
\text { intervention scenario) }\end{array}$ & 957986 & 1042671 & 1127357 \\
\hline $\begin{array}{l}\text { Supply vs. demand gap (stretched } \\
\text { intervention scenario) }\end{array}$ & 894233 & 947598 & 1000963 \\
\hline
\end{tabular}

Table 3.2: Supply and demand gap

EJBE Vol. 5 No. 2/2015 
Forecasting Tourist Arrivals and Supply and Demand Gap Analysis

\section{Findings and Conclusions of the Study 4.1. Findings}

The result shows that among the list of candidate models, model (011) (011) 12 is the most appropriate model to forecast the monthly tourist arrival. Whereas model (210) $(011)_{4}$ is the most appropriate model for forecasting the long term quarterly arrivals to the country. Furthermore, the method has forecasted that the annual tourist arrivals to the country in the year 2015 to be 798,157 and in 2020 the arrivals are expected to rise to 1,130,971, and finally in 2025 to further rise to $1,463,743$. By using the funneling technique, it was possible to get accommodation demands. Accordingly, the demand for accommodation in Addis Ababa is projected to be 580,870 in2015, 822,428 in 2020 and 1,063,987 for the year of 2025 . On the other hand, the supply level is established by considering the existing inventory of hotel rooms as well as considering the number of new hotels which are expected to join the market. In relation to this, the stock of hotel rooms in the city (Addis Ababa) is estimated to be 1,581,509 in 2015, 1,976,529 in 2020 and 2,371,551 in the year of 2025. This, in turn, has helped us to figure out the difference between supply and demand for hotel rooms in the city. The finding shows that the supply of rooms outstrips the demand for the years 2015, 2020 and 2025. In order to test the supply and demand relations under different circumstances a stepped function intervention scenario is used to establish different case scenarios (positive, negative, and starched intervention) which have been investigated to foresee how the relationship between supply and demand of the accommodation sector turns out. In all cases, the supply is found to be much higher than the demand.

\subsection{Conclusion}

Based on the study findings, there is significant gap between the supply and demand conditions on accommodation facility in the city of Addis Ababa. The balance is in favor of big supply of hotel accommodation not commensurable with hotel demand for the coming 5 to 10 years.

This excess supply of hotel accommodation over predicted tourist arrival over the coming ten years has significant implication on the operational performance of the hotels as well as the one`s preparing to enter the market in the future. This is an issue, which requires close follow-up from different stake holders. As a result, it is important to point out that, a lot needs to be done to increase the number of tourist arrivals to Ethiopia. From the forecasted figures, it is clear that the number of visitors to the country as well as the city of Addis Ababa is relatively small in comparison with the potential of the country as well as the supply of accommodation facility in the City.

EJBE Vol. 5 No. 2/2015 
Forecasting Tourist Arrivals and Supply and Demand Gap Analysis

\subsection{Recommendation}

- Based on the supply-demand gap analysis of tourist arrival to hotel accommodation in Addis Ababa City of Ethiopia for the next 10 years, it is recommended that due attention is needed from policy makers, especially from Culture and Tourism Ministry of Ethiopia to promote Ethiopia's tourist sites to attract more tourists to the country.

- It would be more advantageous, if the result figures (the long term forecast of tourist arrival for Addis Ababa, Ethiopia) are verified using qualitative methods probably through use of Delphi method.

- In general extending long term forecast further out is not recommended. Here, the forecast period is extended up to 2025. It is important to highlight that this is done to indicate direction. However, it is assumed that forecast figures up to 2020 are reliable.

- It is important to update the figures by repeating the work by incorporating raw data from years 2013,2014, and 2015 
Forecasting Tourist Arrivals and Supply and Demand Gap Analysis

\section{References}

A.saayman \& M.saayman (2010). Forecasting tourist arrival in south Africa. AddisAbaba Culture \&Tourism Bureau (AACTB).

Addis Ababa investment agency (AAIA)

AHA Apr-June; 2014 Addis Ababa hotel owners trade sectorial association (AHA) quarterly newsletter; vol.1 issue1.

Armstrong, 2001; selecting forecasting methods university of Pennsylvania; Armstrong@ Wharton.upehn.edu.

Buffa \& Sarin, 1987; Modern Production /operation Management; eight edition; John Wiley \& sons Inc.

Christie \& Crompton (2001); Tourism in Africa; Africa Region Working Paper Series No. 12:

Christie et al, 2013; Overview Tourism in Africa: Harnessing Tourism for Growth and Improved Livelihoods, The World Bank: www.worldbank.org/afr/tourism.

Consultant report: Multilink project development solutions; Addis Ababa. Embassy of Japan in Ethiopia :2008 A series of studies on industries in Ethiopia. (Hotel industry in Ethiopia).

FDRE Ministry of Culture \& tourism (tourism development policy Aug 2009).

Frechtling D.C, 2001; Forecasting tourism demand methods and strategies; Butterworth Heinemann.

Gobena \& Gudeta, 2013; Hotel sector investment in Ethiopia Journal of Business management (JBM) Vol.1 (2). P 35-54; Ref 0520130801. GTP plan, 2010; volume I.

H.Song \& G.Li Tourism Demand Modeling and Forecasting A Review of Recent Research H. Song, School of Hotel and Tourism Management The Hong Kong Polytechnic University Hung Hom, Kowloon Hong Kong SAR; Gang Li ,School of Management University of Surrey Guildford GU2 7XH, UK ; epubs.surrey.ac.uk.

IDOWU.k; APanal data Analysis of Demand for Tourism in Africa; Department of Economics, University of Ibadan Nigeria. $14^{\text {th }}$ Africa Economic Society Annual Conference; Cape Town South Africa.

M.Çuhadar, 2014; Modeling and Forecasting in bound Tourism to Istanbul; A comparative analysis; European Journal of Business and Social Sciences, Vol. 2, No.12.

Makridakisetal, 1998; Forecasting methods and application John Wiley and sons Inc.

WBG \& MOCT , 2012; Ethiopia's tourism sector; strategic paths to competitiveness and job creation October 2012; World Bank Group Finance and Private Sector Development \& Ministry of Culture and 
Forecasting Tourist Arrivals and Supply and Demand Gap Analysis

Tourism Federal Democratic Republic of Ethiopia.

MOCT Ministry of Culture and Tourism; Ethiopia A tourist paradise (booklet) www.tourismethiopia.gov.et.

MOCT, 2013; Tourism statistics bulletin 2009-2012 no 10.

MOCT, June 2013; International visitors survey report phase I.

MOFED, 2010; Ministry of Finance and economic development; Growth and Transformation Plan (GTP) 2010/11-2014/15.

Peng Bo , 2012; A Meta-Analysis of International Tourism Demand Elasticity and Forecasting Accuracy; the Hong Kong Polytechnic University School of Hotel and Tourism Managementhttp://www.lib.polyu.edu.hk.

Song et al; Tourism demand modeling and forecasting: how should demand be measured?; Tourism Economics, 2010, 16 (1), 63-81; School of Hotel and Tourism Management, The Hong Kong Polytechnic University, Hung Hom, Kowloon, Hong Kong SAR, PR Chinahmsong@ polyu.edu.hk.

Song, H and G.li ;2010 ;Tourism demand Modeling and forecasting a review recent research

UNWTO \& ETC, 2008; Handbook on Tourism Forecasting Methodologies Copyright ( 2008 World Tourism Organization and European Travel Commission.

UNWTO;2012 Global report on aviation, Am report Volume Five.

UNWTO;2012 Tourism towards 2030,Global overview.

UNWTO;2013 Tourism high light.

UNWTO; 2014 Tourism high light.

UNWTO, 14May 2014; Press release, PR NO: PR14034; Madrid.

UNWTO;2015 World tourism barometer .Volume 13.january 2015.

Wang (2006); Rule induction for forecasting method selection: meta-learning the characteristics of univariate time series; Monash University, Clayton, Victoria 3800, Australia.

Yaffee \& McGree, 1999; Introduction to time series analysis and forecasting with applications of SAS and SPSS academic press, INC.

Z. Ismail et al, 2009; Intervention Model for Analyzing the Impact of Terrorism to Tourism Industry, Journal of Mathematics and Statistics 5(4):322-329.

EJBE Vol. 5 No. 2/2015 